ISSN 0103-9954

\title{
ANÁLISE FAUNÍSTICA DE HIMENÓPTEROS VISITANTES FLORAIS EM FRAGMENTO DE CERRADÃO EM ITIRAPINA, SP $^{1}$
}

\author{
FAUNISTIC ANALYSIS OF FLOWER-VISITING HYMENOPTERA IN CERRADÃO FRAGMENT IN \\ ITIRAPINA, SP
}

Márcia d'Avila ${ }^{2}$ Luis Carlos Marchini ${ }^{3}$

\section{RESUMO}

As abelhas são consideradas os mais eficientes polinizadores. Desse modo, possuem papel indiscutível na reprodução das plantas, aumento da qualidade e quantidade de frutos e sementes e, conseqüentemente, na manutenção dos ecossistemas. O cerrado é um dos ecossistemas mais afetados pelo crescimento das atividades humanas, diminuindo drasticamente sua biodiversidade. A análise faunística além de identificar as espécies e o tamanho das populações, também pode indicar o grau de impacto ambiental numa determinada área. O levantamento dos himenópteros visitantes florais foi realizado quinzenalmente, no período de março de 2003 a fevereiro de 2004, em área de cerradão, com aproximadamente 40 ha, na Estação Experimental de Itirapina, SP. Foram coletados 181 indivíduos, e a família Apidae apresentou maior número de espécies e abundância de indivíduos. As abelhas, Apis mellifera com 55,8\%, seguida de Trigona spinipes com 14,4\% e Exomalopsis (Exomalopsis) sp. com 8,3\% foram as predominantes. Dentre as abelhas coletadas, 30,8\% das espécies são sociais e $69,2 \%$ das espécies são solitárias. Do total de himenópteros coletados, 59,7\% preferiram o período da manhã e 40,3\% o período da tarde para forrageamento e/ou visita. O índice de diversidade de espécies (H) (Shannon-Wiener) foi 1,6933, a variância (H) foi 0,0123 e o índice de uniformidade (E) foi 0,5652, seguindo padrão encontrado em outras áreas de cerrado.

Palavras-chave: cerrado; levantamento; abelhas.

\begin{abstract}
Bees are considered one of the most efficient pollinators. Therefore, they have indisputable role in the plants reproduction, since they increase the quality and quantity of fruit and seeds, and thus they help the ecosystems maintenance. Cerrado is one of the most affected ecosystems due to the growth of human activities, drastically reducing its biodiversity. The faunistic analysis identifies the species and the size of the populations, and can also indicate the degree of environmental impact on a particular area. Surveys on flower-visiting hymenoptera in a "cerradão" area, with 40ha, in the Experimental Station of Itiparina (SP), were conducted every fifteen days from March 2003 to February 2004. From 181 insects collected, the Apidae family was represented by the largest number of species and individuals. The species Apis mellifera (55.8\%), Trigona spinipes (14.4\%) and Exomalopsis (Exomalopsis) sp. (8.3\%) were the most prevalent in the area. Among the bees species collected, $30.8 \%$ were classified as sociable and $69.2 \%$ as solitary. Considering all hymenoptera collected, $59.7 \%$ preferred the morning period and $40.3 \%$ the afternoon period for foraging and/or visiting. The Diversity index (Shannon-Wiener) $\mathrm{H}$ was $1.6933, \mathrm{~V}(\mathrm{H})=0.0123$ and Uniformity index $\mathrm{E}=0.5652$, following pattern found in other areas of cerrado.
\end{abstract}

Keywords: cerrado; survey; bees.

\section{INTRODUÇÃO}

O bioma cerrado é o segundo mais representativo do País, ocupando atualmente 2 milhões de $\mathrm{Km}^{2}$ do território brasileiro. Esse ecossistema se encontra muito fragmentado e degradado pelo avanço das cidades, da agricultura e da pecuária. No estado de São Paulo, o cerrado ocupava $14 \%$ do território no início do século (DURIGAN et al., 2004). Hoje, os remanescentes dessa vegetação cobrem menos de $1 \%$ da área do

1. Parte da Tese, do primeiro autor, apresentada à Escola Superior de Agricultura "Luiz de Queiroz”, Universidade de São Paulo.

2. Engenheira Florestal, Dra., Rua Tuiuti 1840, Apartamento 401, Bairro Centro, CEP 97015-662, Santa Maria (RS). davilamar@hotmail.com

3. Engenheiro Agrônomo, Dr., Professor Titular do Departamento de Entomologia, Fitopatologia e Zoologia Agrícola, Superior de Agricultura "Luiz de Queiroz", Universidade de São Paulo, Av. Pádua Dias 11, Caixa Postal 9, CEP 13418-900, Piracicaba (SP). lcmarchi@esalq.usp.br

Recebido para publicação em 29/06/2006 e aceito em 6/02/2008. 
Estado (248,8 mil $\left.\mathrm{km}^{2}\right)$ e apenas $18 \%$ do que resta se encontra protegido por 32 unidades de conservação e reservas legais (FIORI e FIORAVANTI, 2001).

O cerrado é uma formação típica da zona tropical (PINTO, 1990), com grande biodiversidade, com 4 mil a 10 mil espécies de plantas e cerca de $5 \%$ da fauna mundial, em razão especialmente, da sua grande variedade de paisagens e tipos fisionômicos (FINA, 1999), incluindo vários endemismos (PRIMACK e RODRIGUES, 2001). Apresenta fisionomia variável à medida que a vegetação apresenta maior altura e cobertura, sendo campo, campo-cerrado, cerrado (sensu stricto) e cerradão, que são determinadas pela fertilidade do solo, disponibilidade de água e influência da ocupação humana (EITEN, 1972).

Segundo Bierregaard et al. (1992), a fragmentação dos habitats naturais, em conseqüêncua crescimento das atividades humanas, resulta na formação de fragmentos florestais de diferentes tamanhos e formas, que podem causar o isolamento de populações até a extinção de espécies e, dessa forma, reduzir a biodiversidade local.

De acordo com Dantas et al. (1998), as avaliações da diversidade, tanto da fauna como da flora dos diversos ecossistemas, são de extrema importância para o conhecimento das relações entre os seres vivos, e é o ponto de partida para pesquisas mais específicas.

Os artrópodes correspondem a 75\% dos animais, sendo que destes $89 \%$ são insetos (BUZZI e MIYAZAKI, 1993). Segundo Schoereder (1997), os insetos são adequados para uso em estudos de efeitos de fragmentação florestal e avaliação de impacto ambiental, pois possuem grande diversidade de espécies e de habitats, grande variedade de habilidades para dispersão e seleção de hospedeiros e de respostas à qualidade e quantidade de recursos disponíveis, além de sua dinâmica populacional ser altamente influenciada pela heterogeneidade dentro de um mesmo habitat.

Para estudos em sistemas florestais fragmentados, Didham et al. (1996) consideram mais importantes aqueles capazes de provocar mudanças físicas em seu ambiente e regular a disponibilidade de recursos para outras espécies, tais como polinizadores, predadores de sementes, parasitóides e decompositores.

Muitas espécies de insetos polinizadores são muito susceptíveis à extinção por ocorrerem em densidades populacionais muito baixas e por participarem de interações ecológicas, às vezes, muito estreitas e complexas. Desse modo, a extinção de uma espécie que mantém relações de dependência com outras, pode promover o desaparecimento de várias outras com as quais ela interage (MYERS, 1987).

A diversidade de espécies vegetais em ecossistemas tropicais é muito alta quando comparada com a de outras regiões, e conseqüentemente, o número de insetos visitantes florais e/ou polinizadores também é muito grande, sendo os principais responsáveis pela manutenção da variabilidade genética das plantas, pois promovem a polinização cruzada e, dessa maneira, são essenciais na manutenção dos ecossistemas (SCHLINDWEIN, 2000). A maioria das angiospermas são hermafroditas, ou seja, são autopolinizáveis, mas em razão de alguns mecanismos, como dicogamia, autoincompatibilidade genética e heteromorfia, que impedem a fecundação, a polinização cruzada se faz necessária (RAVEN et al., 2001).

O processo de polinização é realizado pelo vento, chuva, gravidade e por diversos insetos, sendo as abelhas as mais eficientes, tornando-se indispensáveis, pois contribuem para a preservação e perpetuação das espécies vegetais (WIESE, 1995). Desse modo, as abelhas constituem um dos grupos mais importantes para o homem, não somente por permitir a exploração econômica de seus produtos mas sobretudo por contribuir para o aumento da produção de frutos e sementes de diversos vegetais de interesse agroflorestal, que é 143 vezes mais significativo em termos econômicos que suas demais atividades (FREE, 1993; GALLO et al., 2002).

Segundo dados de Silberbauer-Goottsberger e Gottsberger (1988) nos estados de São Paulo, Mato Grosso e Minas Gerais, cerca de 75\% das espécies de plantas do cerrado são polinizadas de forma exclusiva, primária ou secundariamente, por abelhas. Desse modo, a preservação das espécies de abelhas é de suma importância para a manutenção da biodiversidade, visto que para muitas espécies de plantas elas são indispensáveis para que ocorra a polinização (CAMACHO et al., 1999).

As mudanças causadas pelas atividades antrópicas promovem alterações nas condições do habitat, comprometendo a diversidade de abelhas, em conseqüência da destruição de locais usados para nidificação, redução na disponibilidade dos recursos tróficos e eliminação de colônias naturais (MATHESON et al., 1996). 
A diversidade de espécies de um dado local permite avaliar o grau de desgaste dentro de um ecossistema alterado, além de ser um parâmetro relacionado ao equilíbrio dinâmico do ecossistema, por relações estabelecidas entre organismos produtores e consumidores (BROWER e VAN LOON, 1984).

O objetivo deste trabalho foi identificar os insetos visitantes florais da ordem Hymenoptera em uma área de cerradão no estado de São Paulo. Dessa forma, contribuir com informações sobre a composição da fauna de abelhas e de potenciais polinizadores, tanto para determinar padrões das comunidades de abelhas, como possibilitar estratégias futuras de utilização racional da fauna apícola e de conservação das espécies dessa região, bem como servir de base para comparação em pesquisas futuras para avaliar o grau de degradação da biodiversidade de himenópteros.

\section{MATERIAL E MÉTODO}

A pesquisa foi desenvolvida na Estação Experimental de Itirapina, em uma área de cerradão, com área aproximada de 40 ha. A Estação é administrada pelo Instituto Florestal do Estado de São Paulo e está situada nos municípios de Itirapina e Brotas na região central do estado de São Paulo, com área total de 2.300 ha (VEIGA, 1975), sendo 10,9\% da área composta por fragmentos de cerrado e 89,1\% com reflorestamentos de Pinus e Eucalyptus.

As coletas foram realizadas quinzenalmente, no período de março de 2003 a fevereiro de 2004, ao longo de uma trilha pré-existente com aproximadamente $1.500 \mathrm{~m}$ de extensão por $2 \mathrm{~m}$ de largura.

O método de coleta foi baseado no estabelecido por Sakagami e Matsumura (1967). As coletas foram realizadas com rede entomológica das 7 às 18 horas, capturando himenópteros que estivessem visitando as flores. Esse percurso foi realizado duas vezes durante as coletas (manhã e tarde), sendo gastos, aproximadamente, 5 a 10 minutos por planta.

Os insetos coletados foram mortos em éter e colocados em sacos plásticos, sendo anotada a data e o período da coleta. Todo material coletado foi levado ao laboratório e agrupado para posterior identificação, a qual foi realizada por comparação com material existente na coleção de insetos do museu do Setor de Entomologia do Departamento de Entomologia, Fitopatologia e Zoologia Agrícola da Escola Superior de Agricultura "Luiz de Queiroz", Piracicaba.

A análise faunística foi feita de acordo com a metodologia estabelecida por Silveira Neto et al. (1976). A fauna foi caracterizada pelo número de espécies, gêneros e famílias de insetos coletados na trilha.

A posição taxonômica adotada foi baseada em Roig-Alsina e Michener (1993) para as categorias de Família, Subfamília e Tribo. Nos casos em que esses dois últimos táxons não foram referidos pelos autores, seguiu-se o esquema encontrado em Finnamore e Michener (1993) e Griswold et al. (1995). A sistemática para gêneros de Meliponinae foi baseada em Pedro e Camargo (1991).

Os parâmetros faunísticos analisados foram freqüência, constância, dominância, abundância e índice de diversidade.

Freqüência é o percentual do número de indivíduos de cada espécie, em relação ao total de indivíduos coletados, sendo classificados em pouco freqüentes, freqüentes, muito freqüentes e superfreqüentes (SILVEIRA NETO et al., 1976).

A constância foi calculada por meio do percentual de ocorrência das espécies nas coletas (SILVEIRA NETO et al., 1976), e classificadas em constantes, acessórias e acidentais segundo Bodenheimer (1955) citado por Silveira Neto et al. (1976).

Dominância é a capacidade, ou não, da espécie em modificar, em seu benefício, o impacto recebido do ambiente, podendo, desse modo, causar o aparecimento, ou desaparecimento de outros organismos. De acordo com Sakagami e Matsumura (1967), uma espécie é considerada dominante quando seu limite inferior (Li) é maior que o inverso do número total de espécies multiplicado por 100.

Abundância é o número total de espécies amostradas no ambiente, sendo classificadas em rara, dispersa, comum, abundante, muito abundante e super abundante (LUDWIG e REYNOLDS, 1988). As espécies que apresentaram os maiores valores nas classes de freqüência, constância, abundância e dominância foram consideradas como dominantes na área em estudo.

O índice de diversidade utilizado foi o de Shannon-Wiener proposto por Margalef (1951) citado por 
Silveira Neto et al. (1976), por ser um dos melhores para uso em comparações de comunidades, caso não haja interesse em separar os dois componentes da diversidade, abundância e equitabilidade.

A temperatura $\left({ }^{\circ} \mathrm{C}\right)$ e a umidade relativa do ar $(\%)$ foram medidas com um termohigrógrafo digital ao longo da trilha, sendo registrados, no mínimo, três leituras para a realização da média de cada período. Os dados de precipitação foram fornecidos pelos posto Graúna, do município de Itirapina, SP.

\section{RESULTADOS E DISCUSSÃO}

Foram coletados ao total 181 indivíduos pertencentes a seis famílias. A comunidade de abelhas foi representada por 165 indivíduos, ou seja, 91,7\% do total de insetos visitantes florais coletados.

A família Apidae foi a mais representativa, com $85,0 \%$ dos indivíduos coletados, seguida de Halictidae e Vespidae com 5,0\% cada, Pompilidae com 2,2\%, Andrenidae com 1,7\% e Sphecidae com 1,1\%. Somente a espécie Apis mellifera foi responsável por 55,8\% dos indivíduos coletados, seguida de 14,4\% para Trigona spinipes, 8,3\% para Exomalopsis (Exomalopsis) sp., 3,3\% para Centris sp., 2,2\% para Pepsis sp., 1,7\% para Scaptotrigona sp. e Andrenidae, Paratetrapedia sp., Augochlora faxiana, Augochloropsis sp.1, Augochloropsis sp.2, Augochloropsis sp.3, Polistes sp., Polybia sp. e Sphecidae com 1,1\% cada e 0,5\% para o restante das espécies.

A família Halictidae foi a segunda em abundância de espécies com cinco representantes. Campos (1989), Silveira (1989), Pedro (1992), Coelho-Carvalho e Bego (1996), Mateus (1998), Almeida (2002), Andena (2002) e Vieira (2005) também obtiveram resultados similares em áreas de cerrado.

Nos ecossistemas com distúrbios, há uma tendência ao aumento de espécies de Halictidae (ROUBIK, 1989) e a riqueza de espécies tende a aumentar à medida que se afasta do Equador (CARVALHO, 1999).

No geral as espécies coletadas foram, quanto ao parâmetro freqüência, 65,0\% freqüentes, 20,0\% pouco freqüentes, $10,0 \%$ muito freqüentes e 5,0\% super freqüentes; para constância, 85,0\% foram acidentais, 10,0\% acessórias e 5,0\% constantes; para abundância, 65,0\% foram comuns, 20,0\% dispersos, 10,0\% muito abundantes e 5,0\% superabundantes; e para a dominância, 80,0\% foram não dominantes, $15,0 \%$ dominantes e $5,0 \%$ superdominantes.

Entre as espécies dominantes, Apis mellifera foi a única superfreqüente, constante, super abundante e superdominante. Centris sp. foi freqüente, acidental e comum; Exomalopsis (Exomalopsis) sp. e Trigona spinipes foram muito freqüentes, acessórias e muito abundantes (Tabela 1).

Resultado similar foi encontrado por Vieira (2005) em área de cerrado em Cassilândia (MS), onde obteve $25,71 \%$ das espécies dominantes; $42,86 \%$ foram comuns, $11,43 \%$ muito abundantes; $42,86 \%$ freqüentes, $40 \%$ pouco freqüentes e muito freqüentes $11,43 \%$; $45,71 \%$ acidentais, $22,86 \%$ constantes e 31,43\% acessórias.

Almeida (2002) realizou levantamento de abelhas em área de cerradão em Pirassununga (SP) com a mesma metodologia utilizada neste estudo e obteve duzentos indivíduos distribuídos em trinta espécies, sendo Apis mellifera responsável por 79,5\% do total de indivíduos coletados. Nesse caso, pode-se observar que, na área de cerradão de Pirassununga pesquisada por Almeida (2002), o número de espécies de abelhas foi mais que o dobro da área de cerradão de Itirapina, provavelmente, em conseqüência a falta de condições necessárias para manter as espécies nesse fragmento, como a distância do habitat de origem e/ou pela existência de reflorestamentos de Pinus e Eucalyptus muito próximos a área, já que as áreas possuem tamanho similares, 56 ha e 40 ha respectivamente, para Pirassununga e Itirapina.

A temperatura é um dos principais fatores abióticos que influenciam na atividade de vôo das abelhas (IMPERATRIZ-FONSECA et al., 1985). As abelhas preferem o período do dia com temperatura próxima aos $25,0^{\circ} \mathrm{C}$ ou o período com temperaturas mais amenas para forrageamento (SILVEIRA-NETO et al., 1976).

A temperatura média máxima foi de $33,1^{\circ} \mathrm{C}$ no mês de dezembro e a média mínima foi de $18,1^{\circ} \mathrm{C}$ em julho. A maior umidade relativa média foi de $77,4 \%$ em abril e a menor foi $38,2 \%$ em setembro. A precipitação total anual foi de 1654,2 mm, sendo o mês de fevereiro o mais chuvoso, com total de 399,1mm e julho o mais seco, com 5,1 mm (Figura 1). De acordo com dados fornecidos pelo mesmo posto metereológico, o período da pesquisa (2003-2004) apresentou período de estiagem maior que os dois anos anteriores, podendo este fator ter influenciado no florescimento das plantas e conseqüentemente no número 
de himenópteros visitantes florais coletados.

TABELA 1: Classes de freqüência, constância, abundância e dominância dos himenópteros visitantes florais, em área de cerradão, de março de 2003 a fevereiro de 2004, na Estação Experimental de Itirapina, SP.

TABLE 1: Frequency, constancy, abundance and dominance class on flower-visiting hymenoptera, in “cerradão” area, from March 2003 to February 2004, in the Experimental Station of Itirapina, SP.

\begin{tabular}{|c|c|c|c|c|}
\hline \multirow[t]{2}{*}{ Espécies } & \multicolumn{4}{|c|}{ Classes } \\
\hline & Frequência $^{1}$ & Constância $^{2}$ & Abundância $^{3}$ & Dominância $^{4}$ \\
\hline Apis mellifera & $\mathrm{SF}$ & $\mathrm{W}$ & sa & SD \\
\hline Centris sp. & $\mathrm{F}$ & $\mathrm{Z}$ & c & $\mathrm{D}$ \\
\hline Exomalopsis (Exomalopsis) sp. & $\mathrm{MF}$ & Y & $\mathrm{ma}$ & $\mathrm{D}$ \\
\hline Scaptotrigona sp. & $\mathrm{F}$ & $\mathrm{Z}$ & c & ND \\
\hline Tetrapedia rugulosa & $\mathrm{PF}$ & $\mathrm{Z}$ & $\mathrm{d}$ & ND \\
\hline Trigona spinipes & MF & Y & $\mathrm{ma}$ & $\mathrm{D}$ \\
\hline Paratetrapedia sp. & $\mathrm{F}$ & $\mathrm{Z}$ & c & ND \\
\hline Ceretalictus theius & $\mathrm{PF}$ & $\mathrm{Z}$ & $\mathrm{d}$ & ND \\
\hline Augochlora faxiana & $\mathrm{F}$ & $\mathrm{Z}$ & c & ND \\
\hline Augochloropsis sp.1 & $\mathrm{F}$ & $\mathrm{Z}$ & c & ND \\
\hline Augochloropsis sp.2 & $\mathrm{F}$ & $\mathrm{Z}$ & C & ND \\
\hline Augochloropsis sp.3 & $\mathrm{F}$ & $\mathrm{Z}$ & c & ND \\
\hline Andrenidae & $\mathrm{F}$ & $\mathrm{Z}$ & C & ND \\
\hline Mischocyttarus sp. & $\mathrm{F}$ & $\mathrm{Z}$ & c & ND \\
\hline Polistes sp. & $\mathrm{F}$ & $\mathrm{Z}$ & C & ND \\
\hline Polybia sp. & $\mathrm{F}$ & $\mathrm{Z}$ & c & ND \\
\hline Vespidae & $\mathrm{PF}$ & $\mathrm{Z}$ & d & ND \\
\hline Vespidae & $\mathrm{PF}$ & $\mathrm{Z}$ & d & ND \\
\hline Pepsis sp. & $\mathrm{F}$ & $\mathrm{Z}$ & c & ND \\
\hline Sphecidae & $\mathrm{F}$ & $\mathrm{Z}$ & c & ND \\
\hline
\end{tabular}

Em que: ${ }^{1} \mathrm{SF}=$ superfreqüente; $\mathrm{MF}=$ muito freqüente; $\mathrm{F}=$ freqüente; $\mathrm{PF}=$ pouco freqüente; ${ }^{2} \mathrm{~W}=$ constante; $\mathrm{Y}=$ acessória; $\mathrm{Z}=$ acidental; ${ }^{3}$ as $=$ superabundante; ma $=$ muito abundante; $\mathrm{c}=$ comum; $\mathrm{d}=$ disperso; ${ }^{4} \mathrm{SD}=$ superdominante; $\mathrm{D}$ = dominante; $\mathrm{ND}$ = não dominante.

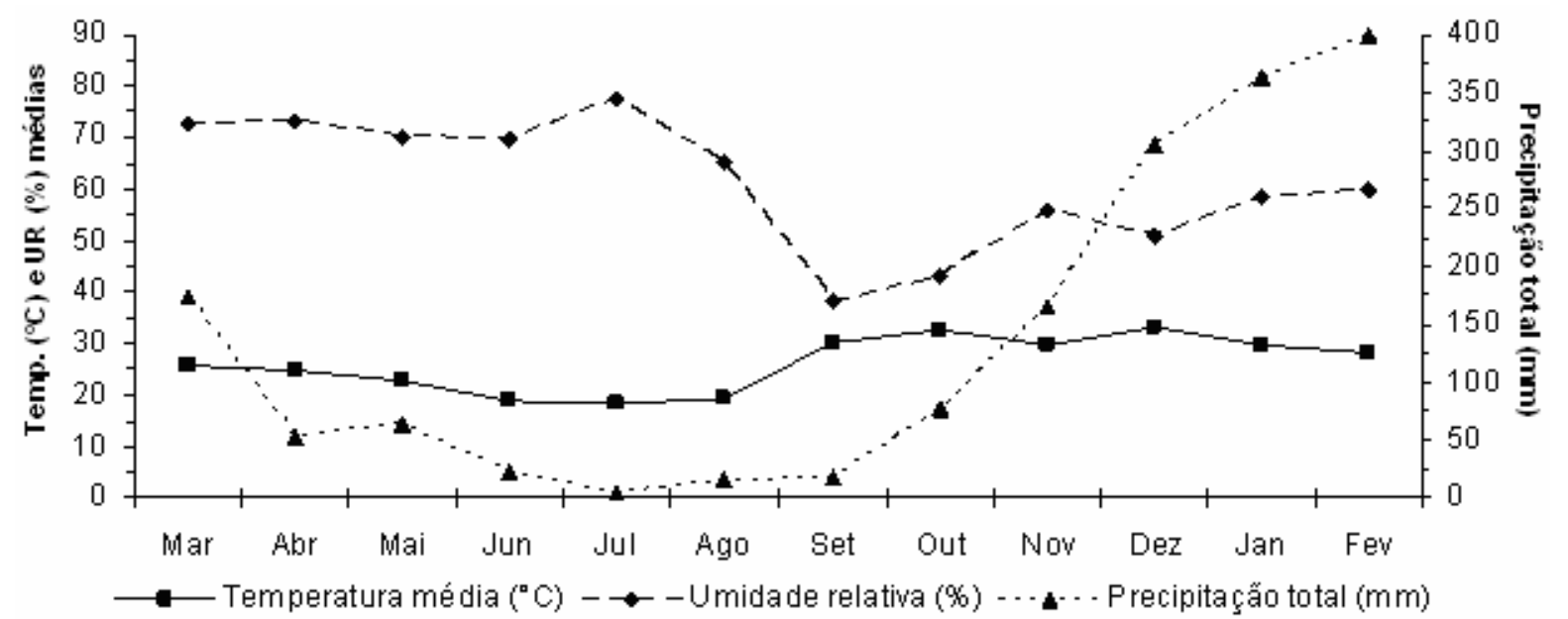

FIGURA 1: Temperatura $\left({ }^{\circ} \mathrm{C}\right)$ e umidade relativa do ar $(\%)$ médias, e precipitação total $(\mathrm{mm})$ mensal, em área de cerradão, de março de 2003 a fevereiro de 2004, na Estação Experimental de Itirapina, Itirapina, SP.

FIGURE 1: Temperature $\left({ }^{\circ} \mathrm{C}\right)$ and relative humidity of the air (\%) average, and total monthly precipitation (mm), in “cerradão" area, from March 2003 to February 2004, in the Experimental Station of Itirapina, SP. 
Segundo Campos (1989), as irregularidades no florescimento, bem como sua duração, levam à ausência de ciclo definido. Isso, sem dúvida, pode exercer uma forte pressão sobre os insetos visitantes, particularmente, as abelhas, dadas às ligações de seus ciclos vitais à floração de diferentes espécies. Quando isso ocorre, a seleção pode favorecer consumidores generalistas, dado que a especiação requer sincronização precisa entre o florescimento e o ciclo reprodutivo da abelha. Essa situação se agrava, quando além de imprevisível, a planta apresenta período de florescimento curto.

Do total de himenópteros coletados, 59,7\% preferiram o período da manhã para forrageamento e/ou visita e 40,3\% preferiram o período da tarde. As espécies da família Apidae também preferiram o período da manhã $(55,8 \%)$ do que o da tarde (44,2\%). A temperatura média do período da manhã foi $26,1^{\circ} \mathrm{C}$, ou seja, com a temperatura mais próxima da ideal. Já o período da tarde teve temperatura média de $28,3^{\circ} \mathrm{C}$.

Os himenópteros foram encontrados visitando flores, praticamente durante todo o ano, em especial de novembro a junho. No período de seca (inverno), as coletas diminuíram muito, provavelmente, em função do número reduzido de plantas floridas (Figura 2).

Pedro (1992) encontrou, para as abelhas não-Apidae, que o número de indivíduos em atividade nas flores foi bem menor durante todo o inverno, quando se observaram temperaturas mais baixas, precipitação pluviométrica quase-ausente, baixos índices de umidade relativa e fotoperíodo mais curto.

A abundância relativa das abelhas, em visita às flores no cerradão de Itirapina, mostrou um padrão fenológico tipicamente tropical, com redução na atividade somente nos períodos mais frios. As espécies eusociais mostraram-se menos suscetíveis às variações de temperatura e umidade relativa do ar.

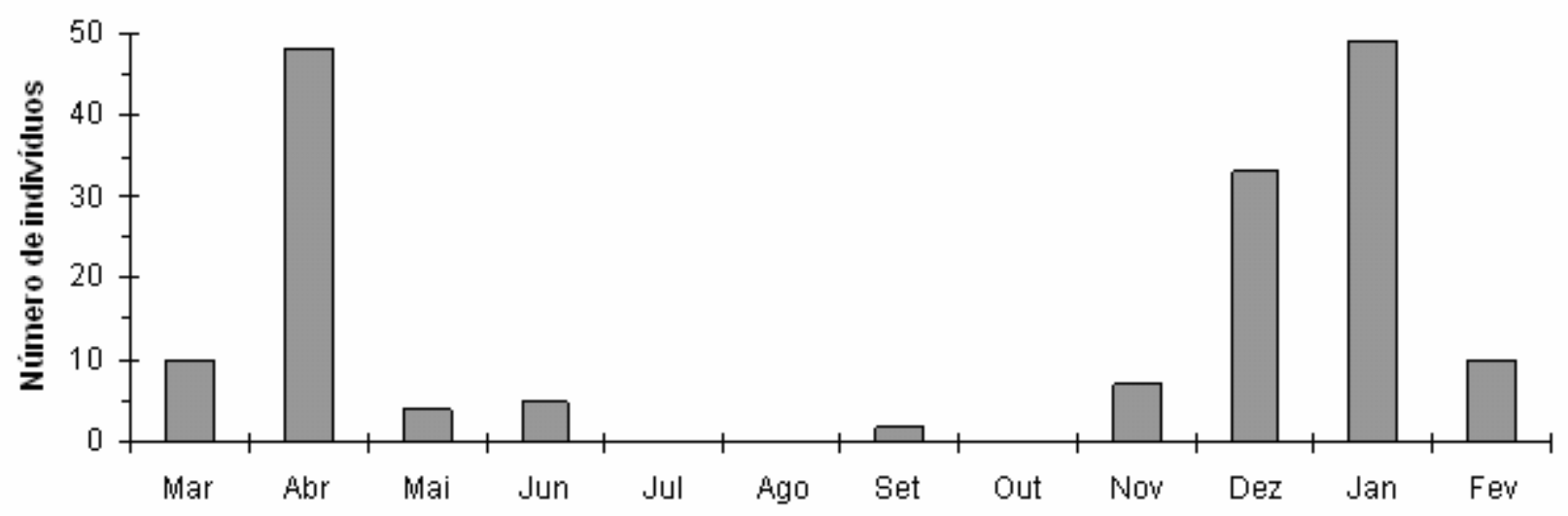

FIGURA 2: Número de abelhas coletadas por mês, em área de cerradão, de março de 2003 a fevereiro de 2004, na Estação Experimental de Itirapina, Itirapina, SP.

FIGURE 2: Number of bees collected per month, in “cerradão” area, from March 2003 to February 2004, in the Experimental Station of Itirapina, SP.

Das espécies de abelhas coletadas, 30,8\% são sociais e representaram 87,3\% de Apoidea e 80,1\% do total de indivíduos; as abelhas solitárias representaram 69,2\% das espécies, sendo 12,7\% de Apoidea e 11,6\% do total de indivíduos. Almeida (2002) encontrou resultado similar, no qual 25,5\% das espécies foram eusociais e representaram $64 \%$ do número total de indivíduos.

As abelhas sociais são na sua maioria generalistas, ou seja, não apresentam preferência por apenas uma ou poucas espécies de plantas para se alimentar, e o número de abelhas generalistas é bem menor quando comparado ao das especialistas, que são aquelas que apresentam preferência por uma ou um número muito pequeno de espécies de plantas. A maioria dos himenópteros e abelhas são especialistas, apresentando maior fidelidade e assim uma polinização mais eficiente, garantindo a perpetuação de muitas espécies (ANTONINI e MARTINS, 2000).

Dentre as espécies sociais dominantes da família Apidae, merece destaque Apis mellifera e Trigona spinipes cuja abundância é explicada por fatores como hábito generalista, largo período de forrageamento, elevada densidade populacional, sofisticado sistema de comunicação e baixa exigência para locais de nidificação (ROUBIK, 1989).

De acordo com vários autores, a família Apidae apresenta maior abundância em relação às demais famílias em área de cerrado, sobretudo as espécies Apis mellifera e Trigona spinipes (BRITO e REGO, 1992; 


\section{MATEUS, 1998; ALMEIDA, 2002; ANDENA, 2002; VIEIRA, 2005).}

Apesar do envolvimento de outros himenópteros, além das abelhas, na visita às flores, quase nunca apareceram como visitantes exclusivos, sendo as abelhas, provavelmente, os polinizadores principais no cerradão.

O índice de diversidade de espécies (H) (Shannon-Wiener) foi 1,6933, Variância $(H)=0,0123$ e $o$ Índice de uniformidade (E) foi 0,5652, seguindo padrão encontrado em outras áreas de cerrado.

Almeida (2002), em estudo de Apoidea, obteve, em área de cerradão, índice de diversidade (Shannon-Wiener) de 2,3987. Outras áreas de cerrado apresentaram os seguintes resultados para índice de diversidade, Corumbataí $\mathrm{H}=1,70$ (CAMPOS, 1989); Paraopeba $\mathrm{H}=1,92$ (SILVEIRA, 1989); Cajuru H = 1,51 (COELHO-CARVALHO e BEGO, 1996); Corumbataí $\mathrm{H}=3,0$ (ANDENA, 2002); Cassilândia $\mathrm{H}=$ 0,8920 (VIEIRA, 2005). De acordo com Silveira Neto et al. (1976) quanto menor o índice de diversidade, maior é a influência dos fatores limitantes e da competição interespecífica no ambiente, apresentando um maior número de espécies comuns e menor de espécies raras.

Apesar de haver similaridade entre o índice de diversidade de espécies dessa pesquisa com o de algumas áreas de cerrado, existe uma grande diferença no número de espécies e de indivíduos coletados nas diferentes áreas de cerrado. Alguns fatores podem ser responsáveis por esses resultados como as características do fragmento, plantios próximos de Eucalyptus e Pinus, e período de estiagem maior no ano do estudo, interferindo no florescimento das plantas.

Segundo Viana et al. (1992) a borda, o tipo de vizinhança, o grau de isolamento e o tamanho efetivo dos fragmentos florestais são os principais fatores que devem ser considerados para medir as alterações nos processos biológicos de um ecossistema. A fragmentação de florestas tem como conseqüência a perda de espécies por meio, especialmente, da destruição do seu habitat; redução do tamanho da população; inibição ou redução da migração; efeito de borda alterando o microclima, sobretudo em fragmentos menores; e eliminação de espécies dependentes de outras já extintas. Espécies raras e com pequena área de distribuição, assim como aquelas que necessitam de habitats muito amplos ou especializados, parecem mais suscetíveis aos efeitos da fragmentação (TURNER, 1996).

Giannotti (1988) e Mendonça (2005) realizaram levantamentos das espécies de plantas na mesma área deste estudo e encontraram um número maior de espécies floridas, podendo ser atribuído a esse fator o período de estiagem mais longo no período da pesquisa, influenciando, dessa forma no menor número de himenópteros coletados.

Muitos fatores podem atuar na determinação da riqueza e abundância das faunas locais de insetos. De acordo com a teoria ecológica, a abundância de alimento favoreceria grandes populações, e a diversidade de fontes alimentares favoreceria maior riqueza de espécies. Desse modo, os resultados dessa pesquisa indicam que o fragmento de cerradão estudado não apresenta abundância nem diversidade de espécies de plantas que servem de alimento para os himenópteros, sobretudo, as abelhas.

\section{CONCLUSÕES}

A comunidade de abelhas encontrada no fragmento de cerradão, aparentemente, não seguiu o padrão geral observado nas comunidades neotropicais, apresentando no total apenas três espécies com muitos indivíduos. A família Apidae foi a mais rica em espécies e a mais abundante.

É importante também ressaltar o baixo número de espécimes e espécies encontradas durante todo o período de levantamento, denotando uma baixa diversidade de himenópteros, como também uma população bastante reduzida em se tratando de uma área protegida de distúrbios antrópicos. Esse dado é um indício de que outros fatores estão interagindo negativamente sobre a diversidade de himenópteros, podendo ser as características do fragmento, como tamanho e grau de isolamento; plantios de espécies exóticas (Pinus e Eucalyptus) em seu entorno, entre outros.

\section{REFERÊNCIAS BIBLIOGRÁFICAS}

ALMEIDA, D. de. Espécies de abelhas (Hymenoptera, Apoidea) e tipificação de méis por elas produzidos em área de cerrado do município de Pirassununga, Estado de São Paulo. Piracicaba, 2002. 103f. Dissertação (Mestrado em Entomologia) - Escola Superior de Agricultura Luiz de Queiroz, Universidade de São Paulo, 2002. 
ANDENA, S.R. A comunidade de abelhas (Hymenoptera: Apoidea) de uma área de cerrado (Corumbataí-SP) e suas visitas às flores. Ribeirão Preto, 2002. 240f. Dissertação (Mestrado em Entomologia) - Faculdade de Filosofia, Ciências e Letras de Ribeirão Preto, Universidade de São Paulo, 2002.

ANTONINI, Y.; MARTINS, R.P. As abelhas e a riqueza nacional. Ciência hoje, São Paulo, v.28, n.164, p.62-63, 2000.

BIERREGAARD, R.O.; LOVEJOY, T.E.; KAPOS, V. et al. The biological dynamics of tropical rainforest fragments. BioSciences, v.42, p.859-866, 1992.

BRITO, C.; RÊGO, M.M.C. Fauna e flora apícola do cerrado, Barreirinhas - MA (Apoidea, Hymenoptera). In: ENCONTRO BRASILEIRO SOBRE BIOLOGIA DE ABELHAS E OUTROS INSETOS SOCIAIS, 1., 1992, Ribeirão Preto. Anais... Ribeirão Preto: Unesp/Rio Claro : USP/FFCL/Ribeirão Preto, 1992. p.128.

BROWER, J.J.; VAN LOON, A.J. Field and laboratory methods for general ecology. 2.ed. Dubuque: Blackwell Science, 1984. 226p.

BUZZI, Z.J.; MIYAZAKI, R.D. Entomologia didática. Curitiba: UFPR, 1993. 262p.

CAMACHO, J.C.B.; MONKS, P.L.; SILVA, J.B. A polinização entomófila na produção e qualidade germinativa de sementes de trevo vesiculoso (Trifolium vesiculosum Savi) cv. EMBRAPA- 28 “Santa Tecla”. Revista Brasileira de Agrociência, Pelotas, v.5, n.2, p.114-119, 1999.

CAMPOS, M.J.O. Estudo das interações entre a comunidade de Apoidea, na procura de recursos alimentares, e a vegetação de cerrado da reserva de Corumbataí, SP. São Carlos, 1989. 114f. Tese (Doutorado em Ecologia) Universidade Federal de São Carlos, 1989.

CARVALHO, C.A.L. de. Diversidade de abelhas (Hymenoptera, Apoidea) no Vale do rio Paraguaçu, município de Castro Alves, Estado da Bahia. Piracicaba, 1999. 104f. Tese (Doutorado em Entomologia) - Escola Superior de Agricultura Luiz de Queiroz, Universidade de São Paulo, 1999.

COELHO-CARVALHO, A.M.; BEGO, L.R. Studies on apoidea fauna of cerrado vegetation at the Panga Ecological Reserve, Uberlândia, MG, Brazil. Revista Brasileira de Entomologia, São Paulo, v.40, n.2, p.147-156, 1996.

DANTAS, J.O.; OLIVEIRA, D.A.; PEREIRA-NETO, A.; et al. Levantamento preliminar de abelhas no Estado de Sergipe. In: CONGRESSO BRASILEIRO DE APICULTURA, 12., Salvador, 1998. Anais... Salvador: Confederação Brasileira de Apicultura, 1998. p.215.

DIDHAM, R.K.; GHAZOUL, J.; STORK, N.E. et al. Insects in fragmented forests: a functional approach. Tree, v.11, n.6, p.255-260, 1996.

DURIGAN, G.; FRANCO, G.A.D.C.; SIQUEIRA, M.F. de. A vegetação dos remanescentes de cerrado no Estado de São Paulo. In: BITENCOURT, M.D.; MENDONÇA, R.R. (Org.). Viabilidade de conservação dos remanescentes de cerrado no Estado de São Paulo. São Paulo: Annablume, 2004. p.29-56.

EITEN, G. The cerrado vegetation of brazil. Botanical Review, Bronx, v. 38, n. 2, p. 2002-341, 1972.

FINA, B.G. Florística e fitossociologia em uma área de cerradão, município de Pirassununga, SP. Rio Claro, 1999. 90f. Dissertação (Mestrado em Entomologia) - Instituto de Biociências, 1999.

FINNAMORE, A.T.; MICHENER, C.D. Superfamily Apoidea. In: GOULET, H.; HUBER, J.T. (Ed.) Hymenoptera of the world: identification guide to families. Ottawa: Canada Communication Group, 1993. p.279-357.

FIORI, A.N.; FIORAVANTI, C. Os caminhos para salvar o cerrado paulista. FAPESP Pesquisa, São Paulo, n.63, p.38-43, 2001.

FREE, J.B. Insect pollination of crops. London: Academic Press, 1993. 684p.

GALLO, D.; NAKANO, O.; SILVEIRA NETO, S.; et al. Entomologia agrícola, Piracicaba: Fealq, 2002. 920p.

GIANNOTTI, E. Composição florística e estrutura fitossociológica da vegetação de cerrado e de transição entre cerrado e mata ciliar da Estação Experimental de Itirapina (SP). Campinas, 1988. 222f. Dissertação (Mestrado em Ecologia) - Universidade de Campinas, 1988.

GRISWOLD, T.; PARKER, F.D.; HANSON, P.E. The bees (Apidae). In: HANSON, P.E.; GAULD, I.D. (Ed.) The hymenoptera of Costa Rica. Oxford: Oxford University Press, 1995. p.650-691.

IMPERATRIZ-FONSECA, V.L.; KLEINERT-GIOVANNINI, A.; PIRES, J.T. Climate variations influence on flight activity of Plebeia remota Holmberg (Hymenoptera, Apidae, Meliponinae). Revista Brasileira de Entomologia, São Paulo, v.29, n.214, p.427-434, 1985.

LUDWIG, J.A.; REYNOLDS, J.F. Statistical Ecology : a primer on methods and computing. New York: John Wiley \& Sons, 1988. 337p. 
MATEUS, S. Abundância relativa, fenologia e visita às flores pelos Apoidea do cerrado da estação ecológica de Jataí - Luiz Antônio - SP. Ribeirão Preto, 1998. 163f. Dissertação (Mestrado em Entomologia) - Universidade de São Paulo, 1998.

MATHESON, A.; BUCHMANN, S.L.; O’TOOLE, C.; WESTRICH, P.; WILLIAMS, I.H. The conservation of bees. London: Academic Press, 1996. 254p.

MENDONÇA, K. Plantas apícolas e caracterização físico-química e polínica de mel produzido por Apis mellifera L. em área de cerrado do município de Itirapina/SP. Piracicaba, 2005. 84f. Dissertação (Mestrado em Entomologia) - Escola Superior de Agricultura Luiz de Queiroz, Universidade de São Paulo, 2005.

MYERS, N. The extinction spasm impending: synergisms at work. Conservation Biology, v.1, n.1, p 14-21, 1987. PEDRO, S.R.M. Sobre as abelhas (Hymenoptera, Apoidea) em um ecossistema de cerrado (Cajurú, NE do estado de São Paulo): composição, fenologia e visita às flores. Ribeirão Preto, 1992. 200f. Dissertação (Mestrado em Entomologia) - Universidade de São Paulo, Ribeirão Preto, 1992.

PEDRO, S.R.M.; CAMARGO, J.M.F. Interactions on floral resources between the Africanized honey bee Apis mellifera L. and the native bee community (Hymenoptera: Apoidea) in a natural "cerrado" ecosystem in southeast Brazil. Apidologie, Versailles, v.22, p.397-415. 1991.

PINTO, M.N. Cerrado: caracterização, ocupação e perpectivas. Brasília - DF: Fundação Universidade de Brasília, 1990. 657p.

PRIMACK, R.B.; RODRIGUES, E. Biologia da conservação. Londrina: E. RODRIGUES, 2001. 328p.

RAVEN, P.H.; EVERT, R.F.; EICHHORN, S.E. Biologia vegetal. Rio de Janeiro: Guanabara Koogan, 2001. 906p.

ROIG-ALSINA, A.; MICHENER, C.D. Studies of the fhylogeny and classification of long-tongued bees (Hymenoptera: Apoidea). The University of Kansas Science Bulletin, Kansas, v.55, p.123-173, 1993.

ROUBIK, D.W. Ecology and natural history of tropical bees. New York: Cambridge University Press, 1989. 514p.

SAKAGAMI, S.F.; MATSUMURA, T. Relative abundance, phenology and flower preference of andrenid bees in Sapporo, north Japan (Hym., Apoidae). Japanese Journal of Ecology, Tokyo, v.17, n.6, p.237-250, 1967.

SCHLINDWEIN, C. A importância de abelhas especializadas na polinização de plantas nativas e conservação do meio ambiente. In: ENCONTRO SOBRE ABELHAS, 4., 2000, Ribeirão Preto. Anais... Ribeirão Preto: Faculdade de Filosofia, Ciências e Letras/USP, 2000. p.131-141.

SCHOEREDER, J.H. Comunidades de formigas: bioindicadores do estresse ambiental em sistemas naturais. In: CONGRESSO BRASILEIRO DE ENTOMOLOGIA, 16., 1997, Salvador. Resumos... Salvador: SEB/EMBRAPACNPMF, 1997. p.233.

SILBERBAUER-GOTTSBERGER, I.; GOTTSBERGER, G. A polinização de plantas do cerrado. Revista Brasileira de Biologia, Rio de Janeiro, v.48, n.4, p.651-663, 1988.

SILVEIRA, F.A. da. Abelhas silvestres (Hymenoptera: Apoidea) e suas fontes de alimento no cerrado da estação florestal de experimentação de Paraopeba - Minas Gerais. Viçosa, 1989. 50f. Dissertação (Mestrado em Entomologia) - Universidade Federal de Viçosa, Viçosa, 1989.

SILVEIRA NETO, S.; NAKANO, O.; VILA NOVA, N.A. Manual de ecologia de insetos. Piracicaba-SP: Ceres, 1976. 419p.

TURNER, I.M. Species loss in fragments of tropical rain forest: a review of the evidence. Journal of Applied Ecology, v.33, p.200-209, 1996.

VEIGA, A.A. Balanços hídricos das dependências da Divisão de Florestas e Estações Experimentais. Boletim Técnico Instituto Florestal, v.18, p.1-34.

VIANA, V.M.; TABANEZ, A.J.A.; MARTINEZ, J.L.A. Restauração e manejo de fragmentos florestais. In: CONGRESSO NACIONAL SOBRE ESSÊNCIAS NATIVAS, 2., 1992, São Paulo. Anais... São Paulo: Instituto Florestal, 1992. p.400-4006.

VIEIRA, G.H. da C. Análise faunística de abelhas (Hymenoptera: Apoidea) e tipificação dos méis produzidos por Apis mellifera L., em área de cerrado no município de Cassilândia/MS. Piracicaba, 2005. 98f. Tese (Doutorado em Entomologia) - Escola Superior de Agricultura Luiz de Queiroz, Universidade de São Paulo, Piracicaba, 2005.

WIESE, H. Novo manual de apicultura. Guaíba: Agropecuária, 1995. 292p. 